\title{
TRANSFER OF A POLARIZED PROTON BEAM FROM AGS TO RHIC
}

\author{
N. Tsoupas*, T. Roser, M. Syphers, A. Luccio, Brookhaven National Laboratory, Upton, NY 11973 \\ D. Underwood Argonne National Laboratory
}

\section{Abstract}

As part of the RHIC project, the RHIC machine will also be able to accelerate polarized proton beam bunches. The bunches will be extracted from the AGS machine, with kinetic energy $\mathrm{T}=25 \mathrm{GeV}$, and transfered into RHIC via the AtR transfer line[1]. When the RHIC machine accelerates polarized protons, it will operate with two full snakes, which define the stable spin direction of a polarized proton beam circulating in each ring, along the vertical. Therefore a polarized proton beam should be injected into the RHIC machine with the stable spin direction along the vertical in order to match that of the RHIC machine. The layout of the dipole magnets of the AtR line[1] creates a dependance, on the injection energy, of the stable spin direction of a polarized proton beam injected into the RHIC machine. In this paper, the study of the stable spin direction (at the RHIC injection point) of a polarized proton beam as a function of the injection energy is presented. A modification of the AtR transfer line, which eliminates this energy dependance (within the range of proton injection energies) of the stable spin direction is also presented.

\section{SECTIONS OF THE ATR LINE AFFECTING THE STABLE SPIN DIRECTION OF A POLARIZED PROTON BEAM}

Two sections of the AtR line are affecting the stable spin direction of a polarized proton beam during injection into the RHIC machine. The first section, which will be referred to as the "12.5 mrad vertical bend", (Fig. 1) is part of the W-line[1] and is located within the $20^{\circ}$ horizontal bend. It starts with the +12.51 mrad vertically pitching magnet which is located midway along the drift space of the second and third combined function magnets[1] and terminates at the exit of the $-12.51 \mathrm{mrad}$ vertically pitching magnet located midway along the drift space of the second and third quadrupoles of the W-line.

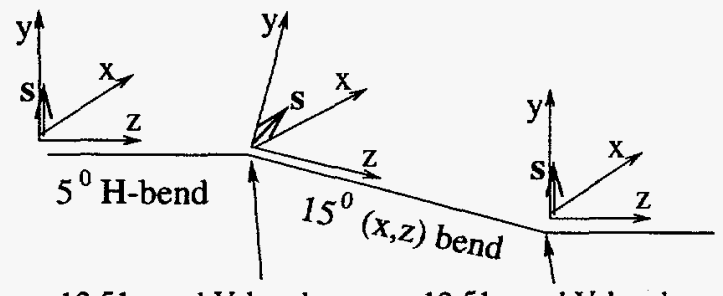

$+12.51 \mathrm{mrad} V$-bend

$-12.51 \mathrm{mrad}$ V-bend

Fig. 1. Diagram of the "12.5 mrad vertical bend".

The second section, which will be referred to as the " 3.0

* Work performed under the auspices of the U.S. Department of Energy mrad vertical bend", (Fig. 2) is located within the injection section[1] of the AtR line and begins with the $3.0 \mathrm{mrad}$ vertically pitching magnet, followed by a Lambertson septum magnet[2], and terminates at the exit of the RHIC injection kicker[3],[4]. It is for the arrangement of the bending magnets in these two vertical bends, that affects the stable spin direction of a polarized proton beam.

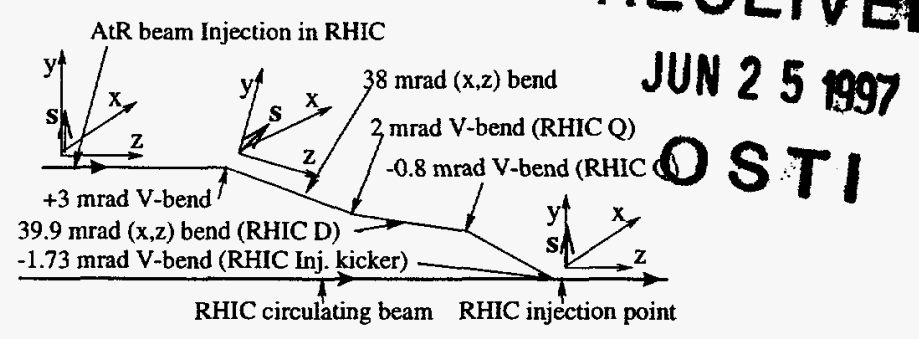

Fig. 2. Schematic diagram of the " 3 mrad vertical bend".

\section{STABLE SPIN DIRECTION AT RHIC INJECTION POINT}

The stable spin direction at the exit of the " 12.5 mrad vertical bend" has been calculated[5] earlier. However the inclusion of the partial snake[6],[7] in AGS as well as the redesign of the injection section of the AtR line[1] required new calculations to determine the stable spin direction at the RHIC injection point. Details of the calculations are also presented in Ref[8]. The new items which are included in the present calculations are:

- A section of the AGS machine (from the center of the straight section C10 of AGS to the HI3 fast extraction point)[8] followed by the AtR line[1].

- The energy dependance of the stable spin direction[7] of a polarized proton beam circulating in the AGS machine.

- The knowledge of the beam ellipsoid in AGS[8].

The calculations to determine the stable spin direction at the "RHIC injection point" were based on the numerical integration of two equations: the differential equation of motion (Eq. 1) of a charged particle moving in static magnetic and electric fields, and the spin precession equation (Eq. 2) of a particle with magnetic moment in the same electric and magnetic field:

$$
\frac{d \mathbf{v}}{d t}=\frac{e}{m \gamma}\left(\mathbf{v} \times \mathbf{B}+\mathbf{E}-\frac{(\mathbf{v} \cdot \mathbf{E}) \mathbf{v}}{c^{2}}\right)
$$

$$
\frac{d \mathbf{s}}{d t}=\frac{e}{m \gamma}\left((G \gamma+1) \mathbf{s} \times \mathbf{B}+(\gamma-1) G \frac{(\mathbf{v} \cdot \mathbf{B})(\mathbf{v} \times \mathbf{s})}{v^{2}}\right)+
$$




$$
+\left(\left(G \gamma+\frac{1}{1+\gamma}\right) \mathbf{s} \times(\mathbf{B} \times v) c^{2}\right)
$$

The simultaneous integration of the (Eq. 1) and (Eq. 2), above, and the required initial conditions which were obtained from the items mentioned above, were performed with a modified version of the RAYTRACE computer code[9] which comes under the name RAYTRACE_SPIN[10].

\section{I Stable Spin Direction at RHIC Injection Point (AGS Partial Snake Off)}

The vertical component $S_{y}$ of the stable spin direction, at the RHIC injection point, is shown in Fig. 3 as a function of $G \gamma$. The empty squares are the $S_{y}$ components of the central trajectory particle. The filled squares are the average vertical spin projection $\left\langle S_{y}\right\rangle$, as calculated from the distribution of the $S_{y}$ component of the 1000 injected particles. The error bars are one standard deviation of the vertical spin projection $S_{y}$ and contains over $90 \%$ of the final spin distribution which is not Gaussian and represent a measure of the spread of the stable spin direction at the RHIC injection point. This spread of the stable spin direction at the RHIC injection point is due to the off central trajectory particles which are experiencing the non vertical fields of the various quadrupoles and combined function magnets (dipole plus quad) of the AtR transfer line. The random sampling of the particles was taken from a beam with a normalized beam emittance of $10 \pi \mathrm{mm}$ mrad for both vertical and horizondal planes, and one standard deviation of the beam momentum spread $\Delta p / p= \pm 0.05 \%$. The calculations performed to obtain the results shown in Fig. 3 assumed that the initial stable spin direction was along the vertical. In an actual injection of a polarized proton beam the assumption that the spin direction is along the vertical will not be valid, at least at the range of energies covered by the Fig. 3. This is because of the AGS partial snake[7], the effect of which is discussed next.

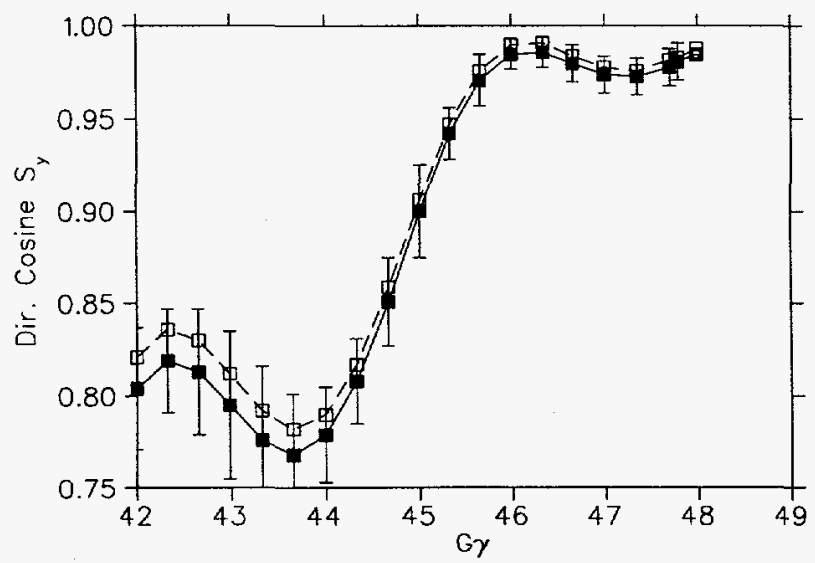

Fig. 3. $S_{y}$ component of the stable spin direction at RHIC injection point. AGS partial snake off.

\subsection{Stable Spin Direction at RHIC Injection Point (AGS Partial Snake On)}

The vertical component $S_{y}$ of the stable spin direction at the RHIC injection point when the partial snake in the AGS is on with $\operatorname{Sin}$ Fig. 4 as a function of the $G \gamma$. The interpretation of the graph's symbols is similar to the one given in the previous subsection. The results of the stable spin direction as presented in Fig. 4 show that the initial stable spin direction, which is defined by the strength of the partial Snake in the AGS, has a profound effect not only in the spread but also on the average value of the stable spin direction itself at the RHIC injection point.

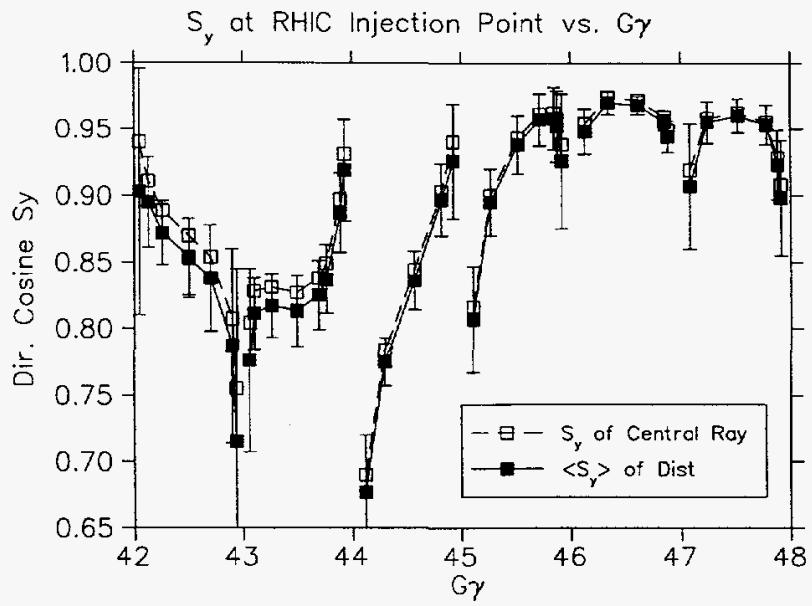

Fig. 4. $S_{y}$ component of the stable spin direction at RHIC injection point. AGS partial snake on.

\section{OPTIMIZATION OF STABLE SPIN DIRECTION WITH INJECTION ENERGY}

The preceeding sections suggest that the optimum energy range for injection of a polarized proton beam into RHIC corresponds to values of $G \gamma$ from $\sim 45$ to 48 . However, it is possible to alter the energy range for optimum polarization transfer of an injected beam into RHIC by employing three additional horizontally bending dipole magnets in the AtR tranfer line. A schematic diagram of the section of the AtR transfer line which includes the three added dipoles, is shown in Fig. 5.

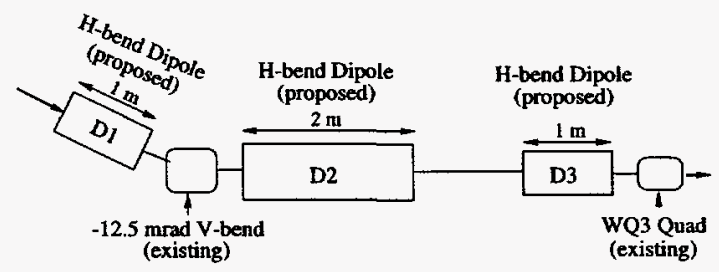

Fig. 5. Diagram of the proposed modification of the AtR transfer line for optimum polarization transfer.

The first of the three proposed dipoles (D1) shown in Fig. 5 is placed in front of the second 12.5 mrad vertically bending magnet of the $20^{\circ}$ bend of the ATR line (see Fig. 1). The second dipole (D2) shown in Fig. 5 is to be placed just after the second 12.5 mrad vertically bending magnet 
with the center of the third dipole (D3) placed downstream from the center of the (D2) dipole, at a distance equal to the distance of the centers of the (D1) and (D2) dipoles. This dipole arrangement is not dispersive, and will only affect the stable spin direction after the vertical magnet shown in Fig. 5. The beam trajectory will be restored at the exit of the D3 dipole, (Fig. 5) and the beam parameters will be practically the same as before the inclusion of the dipoles. The next two subsections present and discuss the results of the stable spin direction at the RHIC injection point when the three horizontal dipoles D1,D2 and D3 shown in Fig. 5 are excited to 1.5 Tesla and bend the beam right,left,right respectively.

\subsection{Stable Spin Direction (AGS Partial Snake Off; AtR Modified)}

With the AtR transfer line modified by the insertion of the three dipoles mentioned above, the calculation for the stable spin direction at the RHIC injection point was repeated. The vertical component $S_{y}$ of the stable spin direction at the RHIC injection point is shown in Fig. 6 as a function of $G \gamma$.

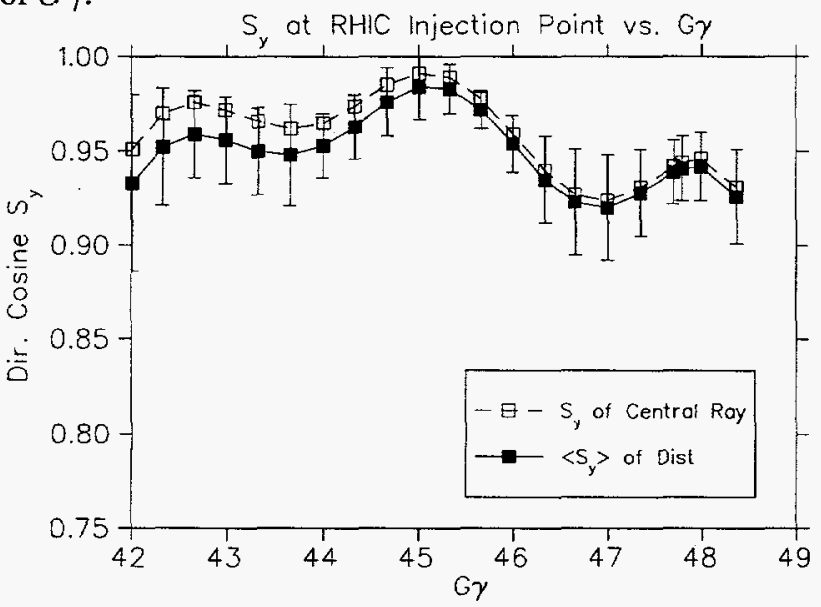

Fig. 6. $S_{y}$ component of the stable spin direction at RHIC injection point. AGS partial snake off, AtR line modified.

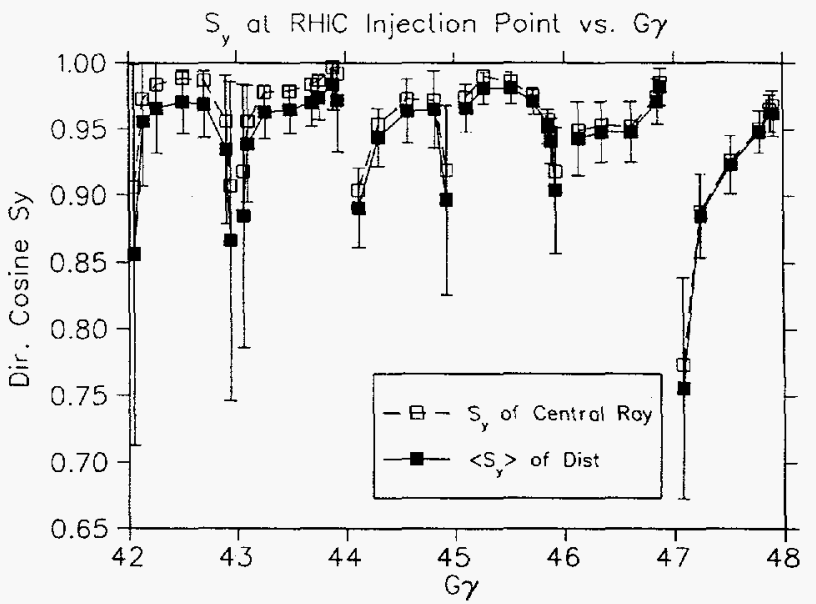

Fig. 7. $S_{y}$ component of the stable spin direction at RHIC injection point. AGS partial snake on, AtR line modified.

\section{REFERENCES}

[1] 'Focusing and matching properties of the AtR transfer line', N. Tsoupas, et, al., BNL, these proceedings.

[2] 'Design and B-Field Measurements of a Lambertson Injection Magnet for the RHIC Machine', N. Tsoupas, E. Rodger, J. Claus, H.W. Foelsche, and P. Wanderer, PAC 1995, p. 1352 (May 1-5 1995), Dallas, TX.

[3] 'The RHIC Injection Kicker', H. Hahn, N. Tsoupas, J.E. Tuozzolo BNL, these proceedings.

[4] 'Beam Injection into RHIC', W. Fischer, H. Hahn, W.W. MacKay, N. Tsoupas, BNL, these proceedings.

[5] S.Y. Lee and E.D. Courant, BNL Technical Note AD/RHIC63.

[6] T. Roser, AIP Conf. Proc. No. 187, p.1442 (1988)

[7] H. Huang et al., Phys. Rev. Lett. 73, 2982 (1994)

[8] 'Closed Orbit Calculations at AGS and Extraction Beam Parameters at H13', N. Tsoupas, H.W. Foelsche, J. Claus, and R. Thern, AD/RHIC/RD-75.

[9] S.B. Kowalski and H.A. Enge, The ion-optical program RAYTRACE NIM A258 (1987) p407.

[10] N. Tsoupas, BNL, private communication

\subsection{Stable Spin Direction (AGS Partial Snake On; AtR Line Modified)}

In these calculations the AtR transfer line is modified as mentioned earlier, and the AGS partial Snake is turned on at a $9^{\circ}$ strength. The vertical component $S_{y}$ of the stable spin direction at the RHIC injection point when the partial Snake in AGS is on, is shown in Fig. 7 as a function of $G \gamma$. 


\section{DISCLAIMER}

This report was prepared as an account of work sponsored by an agency of the United States Government. Neither the United States Government nor any agency thereof, nor any of their emplcyees, makes any warranty, express or implied, or assumes any legal liability or responsibility for the accuracy, completeness, or usefulness of any information, apparatus, product, or process disclosed, or represents that its use would not infringe privately owned rights. Reference herein to any specific commercial product, process, or service by trade name, trademark, manufacturer, or otherwise does not necessarily constitute or imply its endorsement, recommendation, or favoring by the United States Government or any agency thereof. The views and opinions of authors expressed herein do not necessarily state or reflect those of the United States Government or any agency thereof. 


\section{DISCLAMMER}

Portions of this document may be illegible in electronic image products. Images are produced from the best available original document. 